\title{
Effects of stigma, hope and social support on quality of life among Chinese patients diagnosed with oral cancer: A multi-center, cross-sectional study
}

\author{
Ying Zhang \\ China Medical University \\ Chun-Ying Cui \\ China Medical University \\ Yu Wang \\ China Medical University \\ Lie Wang ( $\sim$ liewang@cmu.edu.cn ) \\ China Medical University https://orcid.org/0000-0002-3910-5825
}

Research

Keywords: Oral cancer, Stigma, Hope, Social support, Quality of life

Posted Date: January 6th, 2020

DOl: https://doi.org/10.21203/rs.2.20093/v1

License: (c) (i) This work is licensed under a Creative Commons Attribution 4.0 International License.

Read Full License 


\section{Abstract}

\section{Background}

Improvement of quality of life (QoL) has been one of goals in health care for people living with oral cancer. This study aimed at assessing the QoL and investigate the effects of stigma, hope, social support on QoL among Chinese oral cancer patients.

\section{Methods}

A multiple center cross-sectional study was conducted at the Department of Stomatology, Shengjing Hospital of China Medical University and Stomatology Hospital of China Medical University in Liaoning province, China between May 2016 and October 2017. A total of 230 oral cancer patients were recruited to complete a questionnaire including the Functional Assessment of Cancer Therapy-Head and Neck (FACT-H\&N), the Social Impact Scale (SIS), the Herth Hope Index (HHI) and the Multidimensional Scale of Perceived Social Support (MSPSS). Univariate one-way ANOVA/t-test, Person's $r$ and hierarchical linear regression analysis were conducted to explore the influence factors of $\mathrm{Q} o \mathrm{~L}$ and the relationships between stigma, hope, perceived social support and QoL.

\section{Results}

The mean QoL score was $90.85 \pm 20.15$ among the patients with oral cancer. Stigma was negatively related to QoL, explaining 39.3\% of the variance. In addition, hope and perceived social support were positively associated with QoL, explaining $8.1 \%$ of the variance.

\section{Conclusion}

Overall, Chines patients with oral cancer are suffering from a relatively low level of QoL. Stigma was significantly and negatively associated with QoL, while hope and perceived social support were positively associated with QoL. Oral cancer patients' psychological status should be addressed and adequate intervention based on positive psychological resources should be provided to improve the QoL of patients with oral cancer.

\section{Introduction}

Oral cancer is a broad term for malignant tumors in oral cavity, which may arise as a primary lesion in any part of the oral cavity or oropharynx such as lip, cheek, gingiva, tongue and the floor of mouth [1]. According to World Health Organization (WHO), there were approximately350,000 new cases and 170,000 deaths of oral cancer worldwide in $2018^{[2]}$. Furthermore, it' s estimated by National Central Cancer Registry of China (NCCRC) that oral cancer death accounting for about $1.13 \%$ of all cancer death in China, $2013^{[3]}$. Although incidence and mortality of oral cancer in China were not high, due to the large population basis much attention also should be attracted to oral cancer. Compared with patients with other types of cancer, patients with oral cancer always face substantial challenges including problems 
with speaking, eating, breathing and facial disfigurement, which may significantly impact patient' quality of life (QoL).

QoL is defined by WHO as "individuals' perception of their position in life in the context of culture and the value systems where they live, and in relation to their goals, expectations, standards, and concerns" [4]. Accordingly, QoL for oral cancer patients is a reflection of patients' life status after diagnosed with this disease. Besides, QoL also has become a significant factor in monitoring the treatment and therapeutic procedure success in recently decades ${ }^{[5]}$. Poor QoL of oral cancer patients was always shown to have longer hospital stays, poor postoperative performance, increased narcotic use and complications with treatment, decreased treatment compliance ${ }^{[6]}$. Therefore, researchers are increasingly aware of the value of considering how to improve level of QoL and extend survival length in patients with oral cancer ${ }^{[7]}$. Based on the literature review, we found that not only the general impacts of clinical and demographic differences, but the psychological factors also influence the QoL.

Stigma is defined as "an attribute that is deeply discrediting" or a mark that reduces the sufferer "from a whole and usual person to a trained, discounted one." ${ }^{[8]}$ It has been found to be associated with various types of illness, including but not limited to HIV/AIDS, tuberculosis, and cancers ${ }^{[9]}$. In fact, several studies reported that cancer stigma was prevalent ${ }^{[10]}$. Cancer is recognized as a stigma when it conveys deviation from the normal state. Once individuals are stigmatized, they are often stripped from their social status, and become stereotyped, discriminated, and isolated or even felt exclusion from the society [11]. Stigma in cancer patients was proved to be strongly and consistently associated with negative psychological health, including depressive symptoms ${ }^{[12]}$, anxiety ${ }^{[13]}$, and demoralization ${ }^{[14]}$, which could eventually affect the QoL of patients. Therefore, we supposed that stigma may be an adverse influence factor for the QoL of patients with oral cancer. Besides investigating the risk factors of QoL, more attention should be focused on positive resources in order to improve QoL among patients with oral cancer.

Hope is a positive motivational state which is based on an interactively derived sense of successful agency and pathways. ${ }^{[15]}$. During the treatment, hope could be regarded as one of the most important and effective coping styles when patients face up to the cancer. A systematic review reported that hope was associated with better quality of life and spiritual well-being in Asian patients with cancer ${ }^{[16]}$. In addition to the positive roles of internal psychological construct, the external social resource has been given increasing attention in research on the coping resources of cancer related distress. Social support is generally defined as perceived assistance, esteem and understood one individual receives from families, friends and others ${ }^{[17]}$. Higher levels of social support lead the individuals to believing that themselves are concerned and accepted ${ }^{[18]}$, and it have been associated with positive outcomes, such as improved overall health status, fewer psychological problems and higher QoL ${ }^{[19]}$. A cross-sectional study carried out in Portugal indicates that children with cancer perceived more support form family, they experienced better QoL ${ }^{[20]}$. Chong et al. also demonstrated the higher level of perceived social support among the breast cancer subjects was associated with better QoL in Malaysian [21]. Thus, we hypothesize that the 
presence of hope and supportive interpersonal relationships have the potential to influence the QoL of oral cancer patients in the present study.

However, to the best of our knowledge, extant studies have not yet explored the relationship of patients' QoL with stigma, hope and social support in China. Therefore, in the current study, we aimed to assess the QoL and examined the association between the QoL with stigma, hope and social support in patients with oral cancer.

\section{Methods}

\section{Participants}

The study was approved by the Committee on Human Experimentation of China Medical University, and the study procedures were in accordance with the ethical standards. All participants were recruited from the department of stomatology of Shengjing Hospital of China Medical University and Stomatology Hospital of China Medical University between May 2016 and October 2017, respectively. Once patients agreed to participate in this cross-sectional study, they were distributed questionnaires and informed content to read and sign. Patients with the following inclusion criteria were included into this study: (1) were at least 18 years old, (2) were diagnosed with oral cancers, (3) were after the surgery, (4) were aware of their own diagnosis, (5) were well enough to answer the questionnaires, (6) and had ability to accurately answer questions fluently. Patients with the following criteria were excluded: (1) had psychiatric history or cognition disorders, (2) were illiterate to complete the survey, (3) and had other oral diseases or other cancers. Investigators were responsible for helping them to read and providing explanation without any inducement questionnaire items. Finally, the completed questionnaires were obtained from 230 individuals, including 135 males and 95 females.

\section{Measurement of demographic and clinical variables}

Demographic characteristics consisted of age, gender, marital status, education level, monthly income, work status, residence. Clinical characteristics included reparative therapy, mandibulectomy, distant metastasis, familial inheritance.

\section{Measurement of QoL}

In this study, QoL was measured by the Functional Assessment of Cancer Therapy-Head and Neck (FACT$H \& N)$, it is comprised of 39 questions, and consisted of five dimensions: physical well-being, social/familial well-being, emotional well-being, functional well-being and head and neck cancer specific subscales. Each item was rated on a five-point Likert scale from 0 "not at all" to 4 "very much". The total score ranges from 0 to 144, with the higher score indicating the higher levels of QoL. FACT-H\&N as a specific instrument, has been translated into many languages and validated in previous researches, including Chinese patients ${ }^{[22-25]}$. In our study, the Cronbach's alpha coefficients of the scale was 0.931. 


\section{Measurement of stigma}

The Social Impact Scale (SIS) was developed to assess the level of stigmatization for individuals with cancer or HIV/AIDS ${ }^{[26]}$, and has been applied to different population with good reliability ${ }^{\text {[27] }}$. The 24-item SIS examined 4 domains of stigma: social rejection, financial insecurity, internalized shame, and social isolation. Response to the scale was rated on a 4-point Likert-type scale. Cronbach a coefficients for the scale was 0.947 in this study.

\section{Measurement of hope}

The Herth Hope Index $(\mathrm{HHI})^{[28]}$ was used to assess hope in clinical patients, which contained 12 items and each item had 4 response categories from 1 to 4 . The total score ranges from 12 to 48 , with higher total scores reflected higher level of hope. The Chinese version of $\mathrm{HHI}$ has been demonstrated reliability and validity in cancer patients ${ }^{[29]}$. The Cronbach's a was 0.809 in the sample group.

\section{Measurement of social support}

Social support was assessed using the 12-item version the Multidimensional Scale of Perceived Social Support (MSPSS), which developed by Zimet et al ${ }^{[30]}$. This scale measured perceived social support from three domains: support from family, support from friends and support from significant others. The item is 7-point rating ranging from one "very strongly disagree" to "very strongly agree". The higher total score reflects higher social support. The Chinese version MSPSS was shown to had adequate reliability and validity among other cancer patients ${ }^{[31]}$. The Cronbach's alpha coefficient was 0.928 in this present research.

\section{Statistical analyses}

This study used one-way ANOVA / t-test to describe the mean scores of QoL in different categorical demographic and clinical variables. Pearson's correlation analysis was used to analyze the correlation among QoL, stigma, hope, perceived social support. Hierarchical multiple regression analysis was conducted to investigate the effects of influence factors on QoL. In hierarchical regression analysis, gender, age and potential variables $₫$ which were associated with QoL in univariate analysis $₫$ were entered in step 1. Stigma was added in the step 2. And finally, the independent variables hope and social support were entered into step 3. Indicators provided in the regression models included $R^{2}$, adjust $R^{2}, R^{2}$-change, F-value and standardization regression coefficient $(\beta)$. SPSS for Windows (version 22.0) was used to perform the statistical analyses, with a two-tailed P-value of $<0.05$ considered to be statistically significant.

\section{Results}

\section{Descriptive statistics}


Demographic and clinical characteristics of the patients and the level of the FACT-H\&N scores in different categories of variables were shown in Table 1. There are 230 patients, $135(58.69 \%)$ were men and 95(41.31\%) were women. The age of patients ranges from 18 to 92 years, and the mean (SD) of age was $56.13(13.54)$ years old. $89.13 \%$ of the participants were married or cohabitation, and $145(63 \%)$ who had a household monthly income under 3000 yuan. With regard to clinical variables, only 52(22.6\%) of the patients adopted reparative therapy and $182(79.1 \%)$ of the patients have used marginal mandibulectomy. Of all the variables, scores of FACT-H\&N were found significantly different among different categories of variables including gender $(t=7.060, p=0.008)$, marital status $(t=6.496, p=0.011)$, residence $(t=5.119$, $p=0.025)$, educational background $(F=3.527, p=0.029)$, mandibulectomy $(t=2.644, p=0.009)$, distant metastasis $(t=2.951, p=0.003)$, however, the difference in other variables were not statistically significant.

\section{Correlation between continuous resources and QoL}

Results of correlation analyses between continuous variables were presented in Table 2 . The mean score of QoL among oral cancer patients was $90.85 \pm 20.15$. FACT-H\&N were negatively and significantly correlated with stigma $(r=-0.700, p<0.01)$, and positively and significantly correlated with hope $(r=0.415$, $p \otimes 0.01)$ and perceived social support $(r=0.526, p<0.01)$, respectively.

\section{Hierarchical multiple regression analysis}

As shown in Table 3, all the independent variables that were associated with oral cancer patients' QoL in univariate analysis $(p<0.05)$ were entered into the hierarchical multiple regression model. Each step of independent variables made a significant contribution to the variance of QoL. In step 1, demographic characters including gender, marital status, residence area, education level, and clinical variables including distant metastasis and mandibulectomy as a whole accounted for $9.9 \%$ variance of QoL. In step 2, after controlling for demographic and clinical characteristics, stigma was found to be significantly and negatively associated with QoL. Stigma exhibited significant effect on QoL of oral cancer patients ( $F=26.855$, adjusted $R^{2}=0.504, R^{2}$ - change $=0.393$ ). In step 3, positive psychological resources including hope and perceived social support were added into the model, they are significant positively associated with QoL. The two independent variables accounted for 8.1\% variance of QoL. In addition, all variables in the model could explain $58.5 \%$ of the variance in QoL.

\section{Discussion}

This relatively large-scale multi-center, cross-sectional study of patients with oral cancer was conducted in the northeastern region of china, which was the first study to examine the effects of stigma, hope and social support on QoL in China. Our findings revealed that a majority of Chinese oral cancer patients was suffering from impaired QoL. Mean score of QoL among Chinese patients with oral cancer was $90.85 \pm 20.15$, which is much lower than patients of other countries. Tulio et al. indicated that in Brazil, mean score of FACT-H\&N was $96.6 \pm 20.5^{[32]}$. In Williams' research, the median of FACT-H\&N was 110.20 in America ${ }^{[33]}$. Dominika et al. reported that in Poland, the mean score of FACT-H\&N was 109.19 [34]. 
Despite the fact that there is continual progress in diagnosis and treatment technology and more convenient to obtain quality care in China, our results found that Chinese patients with oral cancer still suffered from poor QoL. Therefore, it's very important to found out essential influencing factors and targeted solutions to improve their QoL.

Among all the demographic and clinical variables, there are some variables related to QoL including marital status, residence area, educational level, undergone mandibulectomy and distant metastasis. These factors in combination explained 9.9\% of the variance in QoL of patients with oral cancer. Findings of our study shows that patients with a college or above educational level had a higher score of QoL, which echoed the findings of Liu's study ${ }^{[35]}$. On the one hand, individuals with higher education level was proved to be positively related to high resilience ${ }^{[36]}$, resilient patients are always considered to possess stronger capabilities to rebound from frustration and tragedy ${ }^{[37]}$. On the other hand, patients with higher education have more access to obtain disease-related knowledge and have a better understanding of their condition. This result indicated that more communications between medical workers and patients are essential for improving patient's QoL. Moreover, as the result showed, patients with distant metastasis had the lowest QoL in this study. However, metastasis is one of the main causes of oral cancer patients' death which is the worst negative events of cancer patients ${ }^{[38]}$. Not only the oral cancer, but metastasis has been confirmed as adverse influential factor in many other cancers in several studies ${ }^{\text {[39-41]. }}$

One of the core findings in this study was that stigma alone explained more than one-third of the variance on QoL. Specifically, stigma was found to be significant negatively associated with QoLðwhich was consistent with previous studies ${ }^{[42,43]}$. Treatment for oral cancer is complex, which can lead to functionality issues such as dysphagia and breathing difficulties, as well as a cosmetic burden with facial disfigurement. However, facial disfigurement always together with the development of shame and a perception of stigma ${ }^{[14]}$. The sense of inner shame plays a crucial role in these patients' self-evaluation processes. High stigma not only has a negative impact on follow-up treatments including treatment compliance, seeking treatment behaviors, self-esteem and social adaptation, but also harmful to patients' recovery which seriously impairing patients' QoL. Noticeably, the mean score of stigmas of the participants in this study were found to be higher than their counterparts in other countries ${ }^{[44-46]}$, which may partly explain why Chinese patients with oral cancer experienced poor QoL. However, to our knowledge there are no other published clinical interventions addressing stigma in oral cancer patients so far ${ }^{[45]}$. Therefore, future studies should focus more on positive psychological factors when stigma impacting oral cancer patients' QoL.

In this study, perceived social support and hope were found to be positively associated with QoL of patients with oral cancer. Patients with high level of hope were likely to experience high QoL that is consistent of the previous study ${ }^{[47]}$. Furthermore, higher level of hope was confirmed to associated with lower rates of alcohol and cigarette abuse, more frequent exercising and better nutrition which were critical factors in preventing cancer recurrence and increasing QoL ${ }^{[48]}$. Hope is a positive coping resource for people experiencing difficult situations. Reasonably, oral cancer patients with higher level of hope 
experienced higher QoL because they are more confident in both daily life and disease conditions. Thus, enhancing the level of hope was one of the important strategies to increase the QoL of patients with oral cancer in China. In addition, we also found that social support was a predictive factor of QoL and positively associated with QoL. Previous study has shown that the absence of social support after diagnosis and during treatment was associated with development of depression and anxiety, and eventually affected the treatment effect ${ }^{[50]}$. However, Li et al. demonstrated that the psychological comfort that cancer patients obtained from family and friends was benefit for clinical treatments and recovery ${ }^{[15]}$. Likewise, Hodges also indicated in their study that social support was positively relate to patients' QoL and promoted well-being, and ultimately improve the QoL ${ }^{[51]}$. Therefore, more social support from family and friends are essential to improve the overall QoL.

The most important value of this study is that we identified the stigma was significant negatively associated with QoL in oral cancer patients. In addition, our study also has added to the evidence that positive resources, hope and social support, were positively associated with QoL in oral cancer patients. Based on our findings, some implications should be mentioned. Firstly, Chinese medical institutions and government should pay more attention to oral cancer patients with low level of QoL. Secondly, it was important for clinicians and nurses to pay more attention to patients with distant metastasis. Thirdly, more attention should be paid to patients with high stigma. There are some studies confirmed that contact with health professionals and the community ${ }^{[52]}$, peer counselling ${ }^{[53]}$, skill building and empowerment ${ }^{[54]}$ were efficient for decrease the stigma. Thus, future research should focus more on longitudinal studies to determine whether reducing stigma is beneficial to improving QoL. Last but not least, some studies have provided the concrete measures and advices to implement psycho-social interventions to enhance QoL in cancer patients. For example, Berg mentioned that hope-based interventions can increase psychological strength, enhance coping with physical symptoms in their research ${ }^{[48]}$. Cramer also indicated in their study that yoga was an effective measure to improving mental health among patients with breast cancer ${ }^{[40]}$. However, up to now, psychological counseling and intervention in clinical setting have not been enough. Overall, health care organizations should realize that the importance of positive psychological strengths for oral cancer patients to combat severe diseases. And more target intervention strategies should be conducted in future researches.

Nonetheless, there were some limitations in our study. First, because of the cross-sectional design and self- reported measures, no causal conclusions could be drawn between psycho-social resources and QoL. Secondly, this study was conducted in one city from a province of the northeastern region of china. Representativeness of sample might be affected. Thirdly, a control group should be included in our study. Thus, the interpretation of the results would be more convincing. Lastly, several potential factors, such as recurrence, pathological stage, may affect QoL of patients with oral cancer, that we didn't included in our study. A lager and longitudinal study should be implied to future researches.

\section{Conclusion}


In conclusion, our study shows that patients with oral cancer in northeastern China are suffering from a relatively low level of QoL. Stigma was significantly and negatively associated with QoL, while hope and perceived social support are positively associated with QoL. Thus, in clinical practice, more attention should be paid to patients' psychological status, and more social support from family, friends or clinical workers should be provided to Chinese oral cancer patients. More importantly, more psychological intervention based on positive psychological resources should be introduced to improve their QoL.

\section{Declarations}

\section{Ethics approval and consent to participate}

All patients provided their informed written consent. This study was approved by the Committee on Human Experimentation of China Medical University.

\section{Consent for publication}

No applicable.

\section{Availability of data and materials}

The datasets analyzed during the current study are available from the corresponding author on reasonable request.

\section{Competing interests}

There were no competing interests.

\section{Funding}

There were no funding sources.

\section{Author's contributions}

YZ carried out data collection, analysis, interpretation, and wrote the paper. CYC performed data collection, clean the data and revised the paper. YW participated in literature search and data collection. LW organized this investigation and supervised the data collection, statistical analysis and paper writing. All authors read and approved the final manuscript.

\section{Acknowledgments}

We would like to thank all the patients who voluntarily participated in this study and research assistants who performed the data collection.

\section{References}


1. Zhang SK, Zheng R, Chen Q, Zhang S, Sun X, Chen W: Oral cancer incidence and mortality in China, 2011. Chin J Cancer Res 2015, 27:44-51.

2. Bray F, Ferlay J, Soerjomataram I, Siegel RL, Torre LA, Jemal A: Global cancer statistics 2018: GLOBOCAN estimates of incidence and mortality worldwide for 36 cancers in 185 countries. CA: A Cancer Journal for Clinicians 2018, 68:394-424.

3. Wanqing, Chen, Rongshou, et al. Cancer incidence and mortality in China, 2013. Cancer Letters, 2017.401:63-71

4. Kuyken W, Orley J, Power M, et al. The World Health Organization quality of life assessment (WHOQOL): Position paper from the World Health Organization. Social Science \& Medicine, 1995, 41(10):1403-1409.

5. Tahani B, Razavi S M, Emami H, et al. Assessment of the quality of life of the patients with treated oral cancer in Iran. Oral and Maxillofacial Surgery, 2017, 21(8):1-9.

6. Zhang, Xing'an, Zhang L, Tan X, et al. Systematic analysis of genes involved in oral cancer metastasis to lymph nodes. Cellular \& Molecular Biology Letters, 2018, 23(1).

7. H Hall P: Book Reviews: STIGMA: NOTES ON THE MANAGEMENT OF SPOILED IDENTITY by Professor Erving Goffman. Published by Pelican Books, 1968. Pp 173. Price: 25p. International Journal of Social Psychiatry 1971, 17:239-239.

8. Neilson K, Pollard A, Boonzaier A, et al. A longitudinal study of distress (depression and anxiety) up to 18 ? months after radiotherapy for head and neck cancer. Psycho-Oncology, 2013, 22(8):18431848.

9. Major B, O'Brien LT: The social psychology of stigma. Annual Review of Psychology 2005, 56:393421.

10. Lebel S, Devins G M. Stigma in cancer patients whose behavior may have contributed to their disease. Future Oncology, 2008, 4(5):717-733.

11. Link BG, Phelan JC: Conceptualizing Stigma. Annual Review of Sociology 2001, 27:363-385.

12. Cataldo J K, Brodsky J L. Lung Cancer Stigma, Anxiety, Depression and Symptom Severity. Oncology, 2013, 85(1):33-40.

13. Brian D. Gonzalez1,2; Paul B. Jacobsen1,2. Depression in lung cancer patients: the role of perceived stigma[J]. Psychooncology.2012,Vol.21(No.3)『239-246.

14. Kissane DW, Patel SG, Baser RE, Bell R, Farberov M, Ostroff JS, Li Y, Singh B, Kraus DH, Shah JP: Preliminary evaluation of the reliability and validity of the Shame and Stigma Scale in head and neck cancer. Head \& Neck 2013, 35:172-183.

15. Dufault K, Martocchio BC: Symposium on compassionate care and the dying experience. Hope: its spheres and dimensions. The Nursing clinics of North America 1985, 20:379-391.

16. Mahendran R, Chua SM, Lim HA, Yee IJ, Tan JYS, Kua EH, Griva K: Biopsychosocial correlates of hope in Asian patients with cancer: a systematic review. BMJ open 2016, 6:e012087-e012087. 
17. Wallston BS, Alagna SW, DeVellis BM, DeVellis RF: Social support and physical health. Health Psychology 1983, 2:367-391.

18. Dreyer J, Schwartz-Attias I: Nursing Care for Adolescents and Young Adults with Cancer: Literature Review. Acta Haematologica 2014, 132:363-374.

19. Ganz PA, Guadagnoli E, Landrum MB, Lash TL, Rakowski W, Silliman RA: Breast cancer in older women: quality of life and psychosocial adjustment in the 15 months after diagnosis. Journal of clinical oncology: official journal of the American Society of Clinical Oncology 2003, 21:4027-4033.

20. Santos S, Crespo C, Canavarro MC, Kazak AE: Family Rituals and Quality of Life in Children with Cancer and Their Parents: The Role of Family Cohesion and Hope. Journal of pediatric psychology 2015, 40:664-671.

21. Ng CG, Mohamed S, See MH, Harun F, Dahlui M, Sulaiman AH, Zainal NZ, Taib NA, My BCCSg: Anxiety, depression, perceived social support and quality of life in Malaysian breast cancer patients: a 1-year prospective study. Health and quality of life outcomes 2015, 13:205-205.

22. Bilal S, Doss J G, Cella D, et al. Quality of life associated factors in head and neck cancer patients in a developing country using the FACT-H\&N. Journal of Cranio-Maxillofacial Surgery, 2015, 43(2):274280.

23. Sobia Bilalab;Jennifer Geraldine Dossab;Simon N. Rogersc. The use of the FACT-H\&N (v4) in clinical settings within a developing country: A mixed method study. J Craniomaxillofac Surg.2014,Vol.42(No.8)『1590-1597.

24. Doss JG, Thomson WM, Drummond BK, Raja Latifah RJ: Validity of the FACT-H\&N (v 4.0) among Malaysian oral cancer patients. Oral Oncology 2011, 47:648-652.

25. Yingbin Yan, Chi Mao, Xin Peng. Usage of FACT-H\&N on measuring quality of life in patients with oral squamous cell carcinoma. Journal of Practical Stomatology. 2010,(5):605-608. (in Chinese)

26. Fife BL, Wright ER: The dimensionality of stigma: a comparison of its impact on the self of persons with HIV/AIDS and cancer. Journal of health and social behavior 2000, 41:50-67.

27. Pan AW, Chung L, Fife BL, Hsiung PC: Evaluation of the psychometrics of the Social Impact Scale: a measure of stigmatization. Int J Rehabil Res 2007, 30:235-238.

28. Herth K: Development and refinement of an instrument to measure hope. Sch Inq Nurs Pract 1991, 5:39-51; discussion 53-36.

29. Yanhua W. Study on feasibility of Chinese version of Herth hope index for cancer patients. Chinese Nursing Research, 2010.

30. Zimet GD, Dahlem NW, Zimet SG, Farley GK: The Multidimensional Scale of Perceived Social Support. Journal of Personality Assessment 1988, 52:30-41.

31. Liu L, Yang YL, Wang ZY, Wu H, Wang Y, Wang L. Prevalence and Positive Correlates of Posttraumatic Stress Disorder Symptoms among Chinese Patients with Hematological Malignancies: A Cross-Sectional Study. Plos one. 2015;10(12):e0145103. 
32. Túlio Eduardo Nogueira, Adorno M , Elismauro Francisco Mendonça, et al. Factors associated with the quality of life of subjects with facial disfigurement due to surgical treatment of head and neck cancer[J]. Medicina oral, patología oral y cirugía bucal. Ed. inglesa, 2018, 23.

33. Williams AM, Lindholm J, Cook D, Siddiqui F, Ghanem TA, Chang SS: Association Between Cognitive Function and Quality of Life in Patients With Head and Neck Cancer. JAMA Otolaryngol Head Neck Surg 2017, 143:1228-1235.

34. Babinska D, Barczynski M, Oseka T, Sledzinski M, Lachinski AJ: Comparison of perioperative stress in patients undergoing thyroid surgery with and without neuromonitoring-a pilot study. Langenbecks Arch Surg 2017, 402:719-725.

35. Liu L, Xu X, Xu N, Wang L: Disease activity, resilience and health-related quality of life in Chinese patients with rheumatoid arthritis: a multi-center, cross-sectional study. Health Qual Life Outcomes 2017, 15:149.

36. Youssef-Morgan CM, Luthans F: Psychological Capital and Well-being. Stress Health 2015, 31:180188.

37. Martínez-Martí ML, Ruch W: Character strengths predict resilience over and above positive affect, self-efficacy, optimism, social support, self-esteem, and life satisfaction. The Journal of Positive Psychology 2017, 12:110-119.

38. Zhang X, Zhang L, Tan X, Lin Y, Han X, Wang H, Ming H, Li Q, Liu K, Feng G: Systematic analysis of genes involved in oral cancer metastasis to lymph nodes. Cell Mol Biol Lett 2018, 23:53.

39. Qiao CX, Zhai XF, Ling CQ, Lang QB, Dong HJ, Liu Q, Li MD: Health-related quality of life evaluated by tumor node metastasis staging system in patients with hepatocellular carcinoma. World $\mathrm{J}$ Gastroenterol 2012, 18:2689-2694.

40. Cramer H, Lauche R, Klose P, Lange S, Langhorst J, Dobos GJ: Yoga for improving health-related quality of life, mental health and cancer-related symptoms in women diagnosed with breast cancer. Cochrane Database Syst Rev 2017, 1: Cd010802.

41. Ahmed AE, Almuzaini AS, Alsadhan MA, Alharbi AG, Almuzaini HS, Ali YZ, Jazieh AR: Health-Related Predictors of Quality of Life in Cancer Patients in Saudi Arabia. J Cancer Educ 2018, 33:1011-1019.

42. Ow CY, Lee BO: Relationships between perceived stigma, coping orientations, self-esteem, and quality of life in patients with schizophrenia. Asia Pac J Public Health 2015, 27:Np1932-1941.

43. Zhang li, yu rui, zheng qiuhua, Yang yu. Relationship model between stigma, family function and quality of life in breast cancer patients. Nursing research,2017,31(11):1333-1336.

44. Cheng CM, Chang CC, Wang JD, Chang KC, Ting SY, Lin CY: Negative Impacts of Self-Stigma on the Quality of Life of Patients in Methadone Maintenance Treatment: The Mediated Roles of Psychological Distress and Social Functioning. Int J Environ Res Public Health 2019, 16.

45. Chambers SK, Baade P, Youl P, Aitken J, Occhipinti S, Vinod S, Valery PC, Garvey G, Fong KM, Ball D, et al: Psychological distress and quality of life in lung cancer: the role of health-related stigma, illness appraisals and social constraints. Psychooncology 2015, 24:1569-1577. 
46. Hasan Shiri F, Mohtashami J, Nasiri M, Manoochehri H, Rohani C: Stigma and Related Factors in Iranian People with Cancer. Asian Pac J Cancer Prev 2018, 19:2285-2290.

47. Stanton AL, Danoff-Burg S, Cameron CL, Bishop M, Collins CA, Kirk SB, Sworowski LA, Twillman R: Emotionally expressive coping predicts psychological and physical adjustment to breast cancer. $\mathrm{J}$ Consult Clin Psychol 2000, 68:875-882.

48. Berg CJ, Vanderpool RC, Getachew B, Payne JB, Johnson MF, Sandridge Y, Bierhoff J, Le L, Johnson R, Weber A, et al: A Hope-Based Intervention to Address Disrupted Goal Pursuits and Quality of Life Among Young Adult Cancer Survivors. J Cancer Educ 2019.

49. Thompson T, Perez M, Kreuter M, Margenthaler J, Colditz G, Jeffe DB: Perceived social support in African American breast cancer patients: Predictors and effects. Soc Sci Med 2017, 192:134-142.

50. Li MY, Yang YL, Liu L, Wang L: Effects of social support, hope and resilience on quality of life among Chinese bladder cancer patients: a cross-sectional study. Health Qual Life Outcomes 2016, 14:73.

51. Hodges K, Winstanley S: Effects of optimism, social support, fighting spirit, cancer worry and internal health locus of control on positive affect in cancer survivors: a path analysis. Stress Health 2012 , 28:408-415.

52. Uys L, Chirwa M, Kohi T, Greeff M, Naidoo J, Makoae L, Dlamini P, Durrheim K, Cuca Y, Holzemer WL: Evaluation of a health setting-based stigma intervention in five African countries. AIDS Patient Care STDS 2009, 23:1059-1066.

53. Lusli M, Peters R, van Brakel W, Zweekhorst M, lancu S, Bunders J, Irwanto, Regeer B: The Impact of a Rights-Based Counselling Intervention to Reduce Stigma in People Affected by Leprosy in Indonesia. PLoS Negl Trop Dis 2016, 10: e0005088.

54. Ebenso B, Ayuba M: 'Money is the vehicle of interaction': insight into social integration of people affected by leprosy in northern Nigeria. Lepr Rev 2010, 81:99-110

\section{Tables}

Table 1 Demographic and clinical characteristics and the score of QoL among oral cancer patients 


\begin{tabular}{|c|c|c|c|c|c|}
\hline \multirow[t]{2}{*}{ variables } & \multirow[t]{2}{*}{ N (\%) } & \multicolumn{4}{|c|}{ FACT-H\&N } \\
\hline & & Mean & $\mathrm{SD}$ & $F / t$ & $P$-value \\
\hline Gender & & & & 7.060 & $0.008^{* *}$ \\
\hline male & $135(58.6)$ & 87.93 & 19.03 & & \\
\hline female & $95(41.4)$ & 95.01 & 21.05 & & \\
\hline Age & & & & 1.289 & 0.199 \\
\hline$<60$ & $139(60.4)$ & 92.42 & 22.32 & & \\
\hline$\geq 60$ & $91(39.6)$ & 88.99 & 17.13 & & \\
\hline Marital status & & & & 6.496 & $0.011^{* *}$ \\
\hline $\begin{array}{l}\text { Single/divorced/ } \\
\text { Separated/widow }\end{array}$ & $25(10.8)$ & 99.44 & 19.07 & & \\
\hline Married/cohabitation & $205(89.2)$ & 89.68 & 20.01 & & \\
\hline Residence & & & & 5.119 & $0.025^{*}$ \\
\hline Rural area & $145(63.1)$ & 93.13 & 19.42 & & \\
\hline Urban area & $85(36.9)$ & 86.96 & 20.87 & & \\
\hline Educational background & & & & 3.597 & $0.029^{*}$ \\
\hline Primary/middle school & $101(43.9)$ & 90.40 & 17.36 & & \\
\hline High / secondary/junior school & $102(44.3)$ & 88.80 & 21.41 & & \\
\hline College or above & $27(11.7)$ & 100.29 & 22.93 & & \\
\hline Household monthly income (yuan) & & & & 1.637 & 0.202 \\
\hline$<3000$ & $145(63.0)$ & 89.93 & 20.58 & & \\
\hline$\geq 3000$ & $85(37.0)$ & 95.62 & 22.02 & & \\
\hline Job status & & & & 1.353 & 0.261 \\
\hline Retirement/unemployed & $133(57.8)$ & 91.31 & 18.71 & & \\
\hline Temporary workers & $32(13.9)$ & 85.48 & 20.42 & & \\
\hline Regular employee & $65(28.2)$ & 93.50 & 22.69 & & \\
\hline Reparative therapy & & & & 1.321 & 0.188 \\
\hline Yes & $52(22.6)$ & 87.61 & 16.92 & & \\
\hline No & $178(77.4)$ & 91.80 & 20.94 & & \\
\hline Mandibulectomy & & & & 2.644 & $0.009^{* * *}$ \\
\hline Yes & $182(79.1)$ & 92.63 & 19.93 & & \\
\hline No & $48(20.9)$ & 84.14 & 19.74 & & \\
\hline Distant metastasis & & & & 2.951 & $0.003^{* * *}$ \\
\hline Yes & $12(5.2)$ & 74.41 & 22.08 & & \\
\hline No & $218(94.8)$ & 91.76 & 19.69 & & \\
\hline Familial inheritance & & & & 1.009 & 0.314 \\
\hline Yes & $15(6.5)$ & 95.93 & 15.07 & & \\
\hline No & $215(93.5)$ & 90.93 & 20.43 & & \\
\hline
\end{tabular}

${ }^{*} p<0.05 \square^{* *} p<0.01 \square^{* * *} p<0.001$; SD standard deviations

Table 2 Scores and correlation of FACT-H\&N with other variables

\begin{tabular}{lllllll}
\hline & Mean & \multicolumn{1}{c}{ SD } & FACT-H\&N & Stigma & Hope & Social support \\
\hline FACT-H\&N & 90.85 & 20.151 & 1 & & & \\
Stigma & 73.47 & 12.550 & $-0.700^{* *}$ & 1 & & \\
Hope & 34.72 & 3.822 & $0.418^{* *}$ & $-0.311^{* *}$ & 1 & \\
Social support & 60.20 & 11.334 & $0.550^{* *}$ & $-0.393^{* *}$ & $0.563^{* *}$ & 1 \\
\hline
\end{tabular}

${ }^{* *} p<0.01 ; \mathrm{SD}$ standard deviations

Table 3 Hierarchical multiple regression analysis results of QoL 


\begin{tabular}{|c|c|c|c|c|c|c|}
\hline \multirow[t]{2}{*}{ Variables } & \multicolumn{2}{|c|}{ Step 1} & \multicolumn{2}{|c|}{ Step 2} & \multicolumn{2}{|c|}{ Step 3} \\
\hline & $\beta$ & $P$-value & $\beta$ & $P$-value & $\beta$ & $P$-value \\
\hline Control variables & & & & & & \\
\hline gender & 0.068 & 0.441 & 0.053 & 0.414 & 0.065 & 0.280 \\
\hline marriage & -0.110 & 0.090 & $-0.128^{* *}$ & 0.008 & -0.086 & 0.055 \\
\hline residence & $-0.176^{*}$ & 0.011 & -0.020 & 0.707 & -0.007 & 0.886 \\
\hline Mandibulectomy & $-0.128^{*}$ & 0.048 & -0.049 & 0.312 & -0.045 & 0.315 \\
\hline Distant metastasis & $-0.160^{* *}$ & 0.007 & -0.037 & 0.445 & 0.029 & 0.552 \\
\hline Edu-1 & 0.052 & 0.449 & -0.076 & 0.144 & -0.059 & 0.218 \\
\hline Edu-2 & 0.004 & 0.954 & -0.071 & 0.183 & -0.076 & 0.116 \\
\hline Stigma & & & $-0.681^{* * *}$ & 0.000 & $-0.571^{* * *}$ & 0.000 \\
\hline hope & & & & & $0.117^{*}$ & 0.031 \\
\hline Social support & & & & & $0.261^{* * *}$ & 0.000 \\
\hline$F$ & $4.145^{* * *}$ & & $26.855^{* * *}$ & & $30.327^{* * *}$ & \\
\hline$R^{2}$ & 0.130 & & 0.523 & & 0.605 & \\
\hline Adjusted $R^{2}$ & 0.099 & & 0.504 & & 0.585 & \\
\hline$R^{2}$-change & 0.130 & & 0.393 & & 0.081 & \\
\hline
\end{tabular}

${ }^{*} p<0.05 \square^{* *} p<0.01 \square^{* * *} p<0.001$

Edu-1 means "primary/middle school" vs. "college or above", Edu-2 means "high/secondary/junior school" vs. "high or secondary school" 